\title{
ON THE EXTENSION OF COMPTON'S EXPERIMENT: A THEORETICAL INVESTIGATION
}

\author{
Amal Pushp \\ Fellow, The Royal Astro. Soc. \\ Burlington House, Piccadilly London W1J 0BQ \\ amalpjha2701@gmail.com
}

September 6, 2019

\begin{abstract}
With new discoveries and insights in atomic and optical physics, the field of spectroscopy is advancing to a new level with applications ranging from material science to astronomy. We here propose a theoretical description of a potential new phenomenon resulting from the extension of Compton's experiment on the scattering of high energy photons through atomic electrons. How the proposed phenomenon can be tested experimentally is discussed in the paper as well.*
\end{abstract}

\footnotetext{
${ }^{*}$ The Idea of the paper is to be presented as part of an International Conference talk (ICECMP-2019)
} 


\section{Introduction}

In 1923, A.H. Compton made a discovery which showed the remarkable transformation that $\mathrm{X}$ rays undergo when they are scattered by atoms $[1,2]$. Some 5 years later, Sir CV Raman, an Indian physicist at the Indian Association for the Cultivation of Science (IACS), discovered along with his students, that this scattering involving the change in wavelength of the radiation is also possible for visible light [3].

Compton, through experimental and theoretical analysis, found the change in wavelength of the scattered $\mathrm{X}$ rays, which is given by,

$$
\delta \lambda=\lambda^{\prime}-\lambda=\frac{h}{m_{e} c}(1-\cos \phi)
$$

\section{Description of the phenomenon}

In this section, we show theoretically that continuos compton scattering of gamma ray (or X ray) photons through atomic electrons produces a new effect in which the Gamma (or X ray) photons transform into extreme UV photons. The angle of scattering between the incident beam of radiation and the atomic electron (in all the individual scatterers) should be maintained at,

$$
\phi=90
$$

At this angle, Compton's equation becomes,

$$
\delta \lambda=\frac{h}{m_{e} c}(1-\cos 90)=\frac{h}{m_{e} c}
$$

This term arises the first time the compton process takes place. Now when the scattered radiation is again allowed to interact with atomic electrons in the scatterer placed at 90 degrees, compton scattering takes place again. If the scattering process continues in this manner, it would result into a self sustained chain until "Beta" is reached (This means only the radiation coming out at 90 degrees goes in a chain and ultimately detected by the detector). It is quite obvious that a point would reach when the radiation energy is very low as compared to the binding energy of electrons of the scatterer and no more shift would occur, here it would be required to use a material with electron binding energy lower than the photon energy or some method to be investigated by the experimenters to cool down the electrons so that photons can further undergo Compton scattering. Finally, photons from the extreme UV region of the electromagnetic spectrum would be detected taking into consideration that the UV Band starts nearly at 124 $\mathrm{eV}$. The value of "Beta" changes with the incident Gamma ray (or X ray) energy.
Therefore, we get,

$$
\delta \lambda_{1}=\frac{h}{m_{e} c}, \delta \lambda_{2}=\frac{h}{m_{e} c}, \ldots ., \delta \lambda_{\beta}=\frac{h}{m_{e} c}
$$

or

$$
\delta \lambda=\delta \lambda_{1}+\delta \lambda_{2}+\ldots+\delta \lambda_{\beta}
$$

or

$$
\delta \lambda=\frac{h}{m_{e} c}+\frac{h}{m_{e} c}+\ldots+\frac{h}{m_{e} c}
$$

or

$$
\delta \lambda=\sum_{i=1}^{\beta} \frac{h}{m_{e} c}
$$

Now the wavelength of incident radiation in angstroms is given by,

$$
\lambda=\frac{12.398}{E_{\gamma}(K e V)}
$$

We get the total shift in wavelength i.e. from Gamma ray (or X ray) to extreme ultraviolet of the form,

$$
\delta \lambda=\frac{12.398}{E_{\gamma}(K e V)}+\sum_{i=1}^{\beta} \frac{h}{m_{e} c}
$$

i.e. [wavelength of incident radiation in $\AA+$ shift obtained by continuos compton scattering at 90 degrees till "Beta"(also in $\AA$ )]. Thus the above equation represents the proposed phenomenon and will give the wavelength of UV photons in Angstroms. Now, this effect is important as it shows that simply through extending the compton process, there is a transformation from one type of EM radiation to another type in the electromagnetic spectrum. Usually, transformation from one type of radiation to another type involves a series of complex processes and apparatus like the one proposed by J.M. Cole et al. in their paper [4]. Their paper reported generation of hard photons (gamma) from an all-optical Inverse Compton scattering situation. More specifically, from infrared to gamma radiation. Another difference which can be seen here is that they discovered transformation from lower energy photons to higher energy made possible by Inverse Compton process and we have predicted an effect which would undergo transformation from high energy photons to lower energy photons in the extreme ultraviolet region which is done simply by extending the Compton effect.

In the next section we look on to the theoretical calculations (of wavelengths of UV photons, value of Beta for varying radiation energies etc) and plot graphs for the same. 
SEPTEMBER 6, 2019

\section{Theoretical Results and Graphs}

Here, the value of "Beta" is found through trial and error and the value of the Compton wavelength and the obtained wavelength of UV photons is expressed in $\AA$. Therefore, from the equation we derived in the previous section, we get for,

$$
\begin{gathered}
E_{\gamma}=100 \mathrm{KeV} \\
\delta \lambda=\frac{12.398}{100}+\sum_{i=1}^{4110} 0.0243
\end{gathered}
$$

or

$$
\delta \lambda=0.12398+99.873
$$

or

$$
\delta \lambda=99.997
$$

We calculated the energy of the UV photons using the online Angstrom - ElectronVolt (eV) Converter [6]. Therefore we found the energy of the UV photons (for $100 \mathrm{KeV}$ incident Gamma Ray energy) as $123.98 \mathrm{eV}$. Hence we summarise our results in the first table.

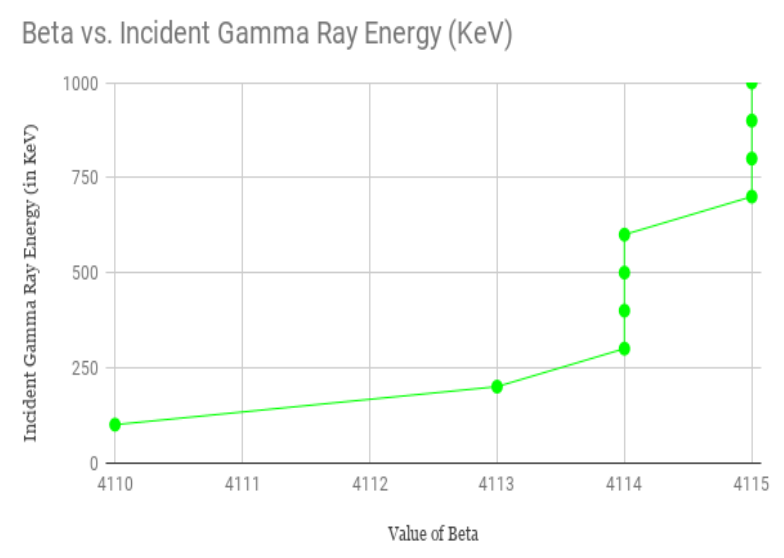

Incident Gamma Ray Energy (in KeV) Vs. Predicted EUV Photon Energy (in eV)

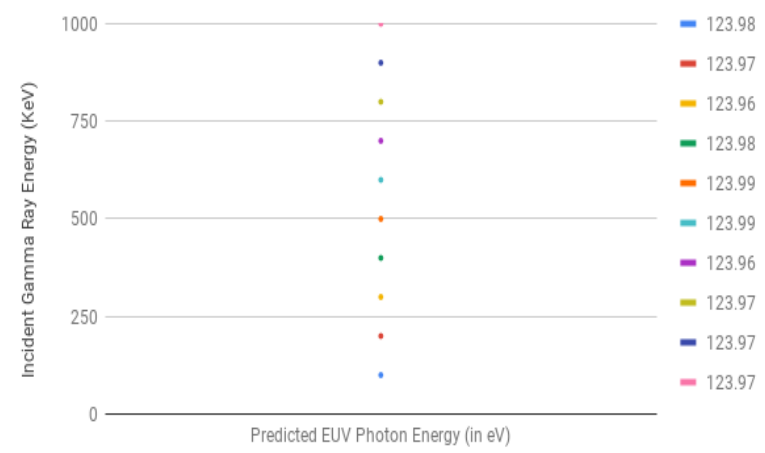

\section{Beta vs. Predicted EUV Photon Energy (in eV)}

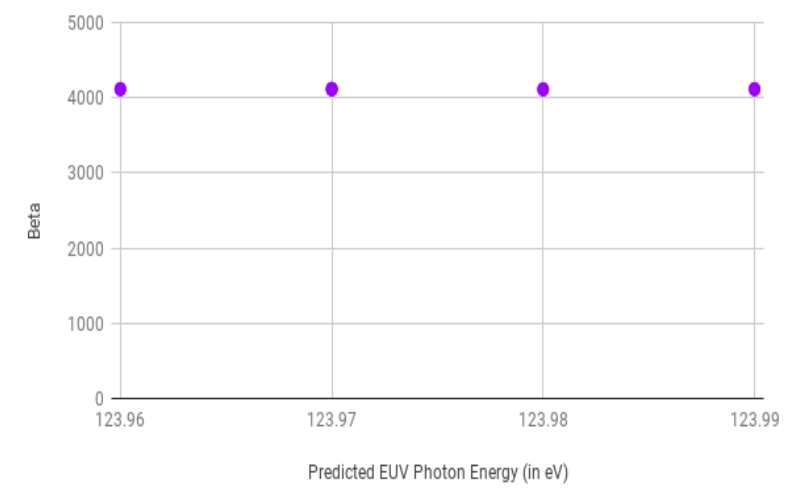

The Previous Table of results was obtained with regard to Gamma Radiation. Now we find results taking X Rays into consideration. Therefore, we get using our equation for,

$$
\begin{gathered}
E_{X \text { Rays }}=0.2 \mathrm{KeV} \\
\delta \lambda=\frac{12.398}{0.2}+\sum_{i=1}^{1564} 0.0243
\end{gathered}
$$

or

$$
\delta \lambda=62+38.0052
$$

or

$$
\delta \lambda=100.005
$$

and obtained wavelength of UV photons is $123.97 \mathrm{eV}$. Further Results are summarised in table 2. It is to be noted here that we have assumed that $\mathrm{X}$ ray band starts at $0.2 \mathrm{KeV}$ (specifically it is the soft $\mathrm{X}$ ray regime) for convenience in graphs.

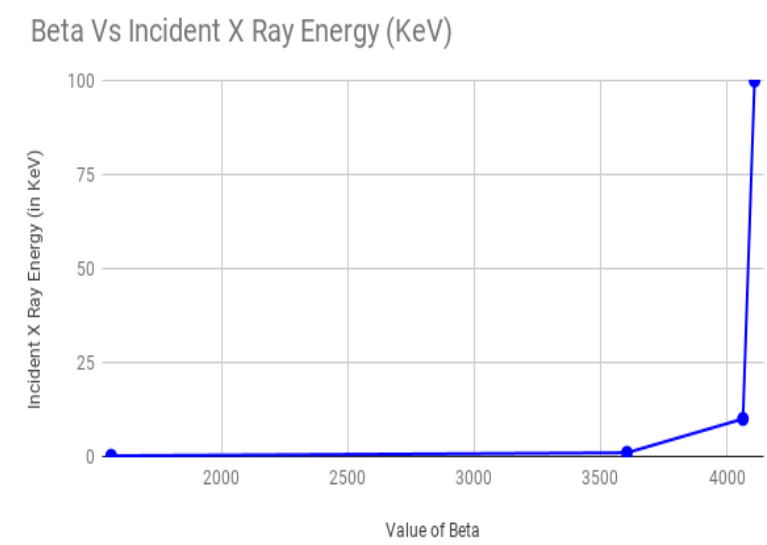




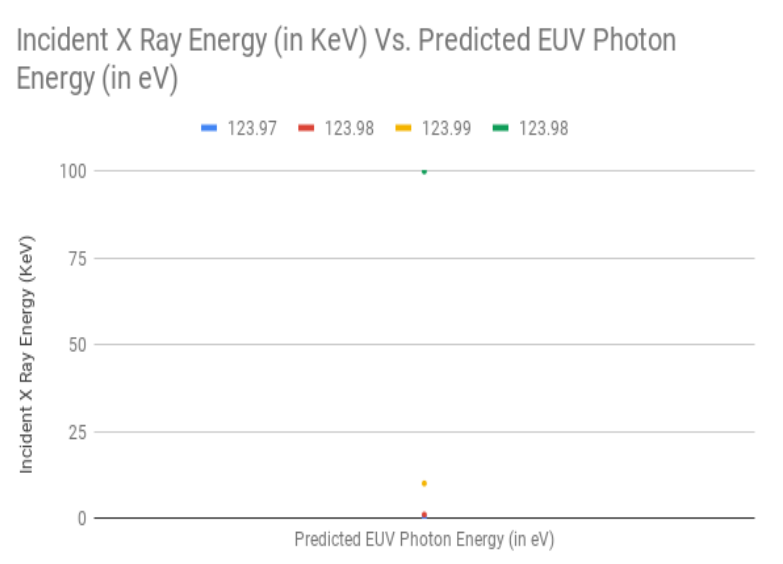

\section{Beta Vs. Predicted EUV Photon Energy (in eV)}

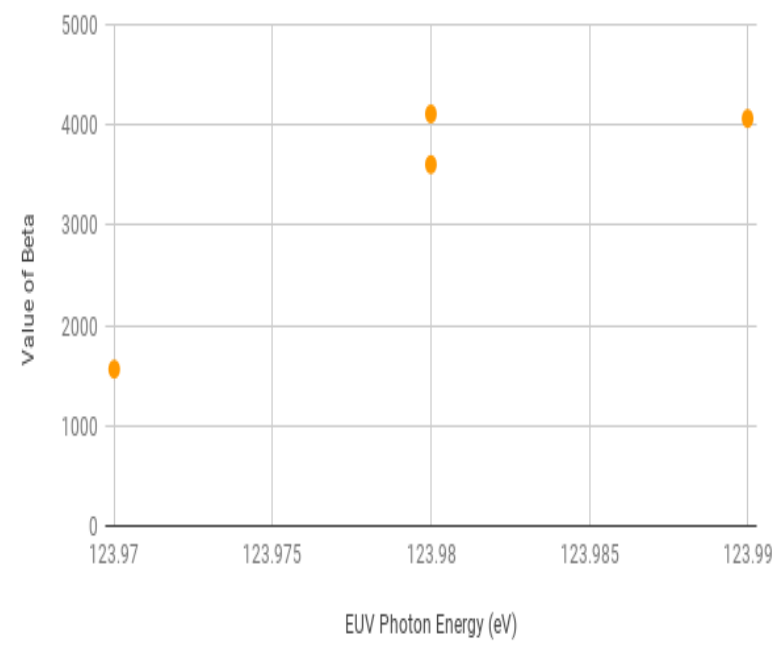

\section{Proposed Experimental Setup}

In this section we propose the experimental setup and apparatus which can be undertaken to verify our theoretical prediction and results. It would be wise to note here that more energetic photons experience a larger percent loss in energy in Compton scattering. This becomes clear from the expression for fractional loss in energy [6]. It is given simply by,

$$
\frac{K}{E}=\frac{h c \Delta \lambda / \lambda(\lambda+\Delta \lambda)}{h c / \lambda}=\frac{\Delta \lambda}{\lambda+\Delta \lambda}
$$

The Experimental setup would include a source of high energy radiation (Gamma Rays or X rays in particular cases), an apparatus consisting of several compton scatterers arranged in a chain (right angles to each other and according to the principles of the proposed phenomenon). The scatterer should be of a material consisting of free electrons and having electron binding energy lower than the energy of the incident electromagnetic radiation (for eg. $2 p$ electron of Carbon has binding energy nearly equal to $11.26 \mathrm{eV}$ and that of $4 \mathrm{p}$ Germanium is $8 \mathrm{eV}$ and so on). The radiation emitted out of the last scatterer (the value "Beta") is allowed to pass through the collimators and then allowed to fall onto the photon detector combined with the spectrum analyzer to recognise the extreme ultraviolet (EUV) photons.

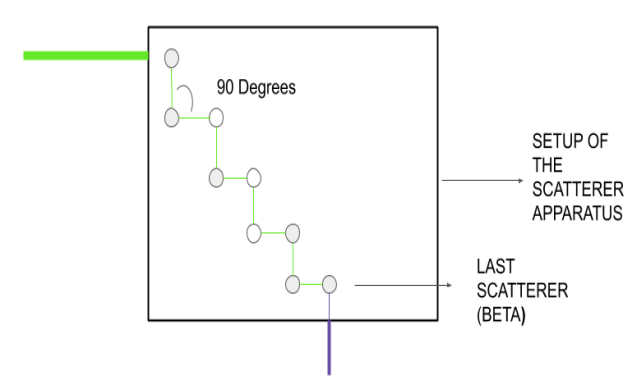

\section{Conclusion}

Discovery of the Compton effect proved that electromagnetic radiation can be treated as a stream of particles which follow the laws of mechanics. Compton effect generally means that High energy photons when scattered, transfer part of their energy and momentum to the particles off which they are scattered which results into increase in wavelength of the radiation but they still remain in the same part of the EM spectrum whereas through inverse compton scattering, photons from the very low energy ranges can acquire a boost in energy resulting into transformation from one type of EM radiation to another. But our research and theoretical analysis has shown that even through the former (basic Compton scattering), photons can be transformed from one type to another type in the EM spectrum. Thus Our proposal can be concluded as: "There is a transformation in Gamma Rays (or X Rays) upon continuos Compton scattering through atomic electrons at 90 degrees, in a way that they result into photons from the extreme ultraviolet region of the electromagnetic spectrum". Earlier, discoveries like these (for eg. the Compton, Raman, Cerenkov and Mossbauer effects etc) have been found to be extremely useful in spectroscopic studies. So upon experimental confirmation of the prediction, it is likely to be significant for the same. This effect is unique in its own way as there are no complex processes involved and it is an extension of the Compton effect. Though the experimental confirmation 
would be challenging for the experimenters, still the effect proposed is in full agreement with the basic principles and provided the necessary conditions, results can be tested with experiment in a laboratory.

\section{Acknowledgments}

I acknowledge my colleague Akruti Mishra for useful discussions during the early periods of this work. I specially thank my elder brother Mr. Ujjwal Pushp for technical assistance and Mr. Chandan Roy for encouragement. I am highly grateful to Prof J. Christian and Prof. Partha Ghose for their valuable suggestions. I also take this opportunity to acknowledge the Royal Astronomical Society, London for electing me a Fellow and Sir M.J. Rees for his nomination.

\section{References}

[1] A. H. Compton. A Quantum Theory of the Scattering of X-Rays by Light Elements, Physical Review. 21 (5), 483-502 (1923).
[2] A. H. Compton. Nobel lecture: X-Rays as a Branch of Optics (1927).

[3] Raman, C.V.; Krishnan, K.S. A New Type of Secondary Radiation, Nature. 121, 501 (1928).

[4] J.M. Cole, K.T. Behm, E. Gerstmayr, T.G. Blackburn, J.C. Wood, C.D. Baird, M.J. Duff, C. Harvey, A. Ilderton, A.S. Joglekar, K. Krushelnick, S.Kuschel, M. Marklund, P. Mckenna, C.D. Murphy, K. Poder, C.P. Ridgers, G.M. Samarin, G. Sarri, D.R. Symes, A.G.R. Thomas, J. Warwick, M. Zepf, Z. Najmudin, Stuart P.D. Mangles et al. Experimental Evidence of a Radiation Reaction in the Collision of a High-Intensity Laser Pulse with a Laser- Wakefield Accelerated Electron Beam., Phys. Rev. X, 8, 011020 (2018). arXiv e-print arXiv: 1707.06821 [physics.plasma-ph].

[5] Eisberg, R.M.; Resnick, R. Quantum Physics of Atoms, Molecules, Solids, Nuclei and Particles., New York: Wiley (1974).

[6] https://people.mbi.ucla.edu/sumchan/crystallography/angeV_convertor.html.

Table 1:

\begin{tabular}{||cccc||}
\hline Incident Gamma Energy (in KeV) & Value of Beta & Energy of UV Photons (in eV) & Wavelength of UV (in $\AA$ ) \\
\hline \hline 100 & 4110 & 123.98 & 99.997 \\
\hline 200 & 4113 & 123.97 & 100.0079 \\
\hline 300 & 4114 & 123.96 & 100.0115 \\
\hline 400 & 4114 & 123.98 & 99.995 \\
\hline 500 & 4114 & 123.99 & 99.9908 \\
\hline 600 & 4114 & 123.99 & 100.0115 \\
\hline 700 & 4115 & 123.96 & 100.0095 \\
\hline 800 & 4115 & 123.97 & 100.0082 \\
\hline 100 & 4115 & 123.97 & 100.0069 \\
\hline
\end{tabular}

Table 2:

\begin{tabular}{||cccc||}
\hline Incident X Ray energy (in KeV) & Value of Beta & Energy of UV Photons (in eV) & Wavelength of UV (in $\AA$ ) \\
\hline \hline 0.2 & 1564 & 123.97 & 100.005 \\
\hline 1 & 3605 & 123.98 & 99.9995 \\
\hline 10 & 4064 & 123.99 & 99.995 \\
\hline 100 & 4110 & 123.98 & 99.997 \\
\hline
\end{tabular}


SEPTEMBER 6, 2019

\section{Appendix}

We derived the total shift in wavelength from Gamma Ray to EUV as given by eq. (7). For a more rigorous approach we can integrate equation (7) in order to yield the overall shift from a particular scatterer system. This is done as below,

$$
\delta \lambda=\frac{12.398}{E_{\gamma}(\mathrm{KeV})}+\sum_{i=1}^{\beta} \frac{h}{m_{e} c}
$$

Taking integral at $\delta \lambda$ from 0 to $\lambda$, we get,

$$
\int_{0}^{\lambda} \delta \lambda=\frac{12.398}{E_{\gamma}(K e V)}+\int \sum_{i=1}^{\beta} \frac{h}{m_{e} c}
$$

or,

$$
[\delta \lambda]_{0}^{\lambda}=\frac{12.398}{E_{\gamma}(K e V)}+\int \sum_{i=1}^{\beta} \frac{h}{m_{e} c}
$$

Simplifying the above equation we thus get an expression of the form,

$$
\lambda=\frac{12.398}{E_{\gamma}(K e V)}+\int \sum_{i=1}^{\beta} \frac{h}{m_{e} c}
$$

It should be noted here that we have not integrated eq. (6) here since it gives the wavelength of the incident radiation. 